\title{
Selection, Subdivision and Extinction and Recolonization
}

\author{
Joshua L. Cherry ${ }^{1}$ \\ National Center for Biotechnology Information, National Library of Medicine, National Institutes of Health, Bethesda, Maryland 20894
}

Manuscript received April 14, 2003

Accepted for publication November 2, 2003

\begin{abstract}
In a subdivided population, the interaction between natural selection and stochastic change in allele frequency is affected by the occurrence of local extinction and subsequent recolonization. The relative importance of selection can be diminished by this additional source of stochastic change in allele frequency. Results are presented for subdivided populations with extinction and recolonization where there is more than one founding allele after extinction, where these may tend to come from the same source deme, where the number of founding alleles is variable or the founders make unequal contributions, and where there is dominance for fitness or local frequency dependence. The behavior of a selected allele in a subdivided population is in all these situations approximately the same as that of an allele with different selection parameters in an unstructured population with a different size. The magnitude of the quantity $N_{\mathrm{e}} s_{\mathrm{e}}$, which determines fixation probability in the case of genic selection, is always decreased by extinction and recolonization, so that deleterious alleles are more likely to fix and advantageous alleles less likely to do so. The importance of dominance or frequency dependence is also altered by extinction and recolonization. Computer simulations confirm that the theoretical predictions of both fixation probabilities and mean times to fixation are good approximations.
\end{abstract}

$\mathrm{P}_{\mathrm{s}}$ OPUlation subdivision has many populationgenetic consequences. The amount of neutral variation maintained in a population, the distribution of coalescence times, expected times to fixation or loss of alleles, and fixation probabilities of selected alleles can all be altered by subdivision. An important determinant of the magnitudes, and even the directions, of these effects is whether gene flow among subpopulations includes local extinction and subsequent recolonization.

In the absence of extinction and recolonization, subdivision increases the amount of standing neutral variation and lengthens coalescence times. By these measures subdivision therefore increases effective population size, $N_{\mathrm{e}}$. Extinction and recolonization can diminish and even reverse these effects (Slatkin 1977; Maruyama and Kimura 1980), so that effective size can be either larger or smaller than actual size.

The simplest case involving natural selection is genic selection in the absence of extinction and recolonization. Under these conditions, so long as migration is symmetric, subdivision has no effect on the fixation probability of an allele under selection (MARUyama $1970,1974)$. This would seem to suggest that for the purpose of understanding the behavior of selected alleles $N_{\mathrm{e}}$ is unaffected by subdivision, since fixation probability is generally thought of as a function of $N_{\mathrm{e}} s$, where $s$ is the selection coefficient. The apparent discrepancy

\footnotetext{
${ }^{1}$ Address for correspondence: National Center for Biotechnology Information, National Library of Medicine, National Institutes of Health, 45 Center Dr., Bethesda, MD 20894. E-mail: jcherry@ncbi.nlm.nih.gov

between the $N_{\mathrm{e}}$ that applies to fixation probabilities of selected alleles and the $N_{\mathrm{e}}$ describing the behavior of neutral alleles can in some cases be resolved with the notion of the effective selection coefficient $s_{\mathrm{e}}$ (CHERry and Wakeley 2003). $N_{\mathrm{e}} s_{\mathrm{e}}$, rather than $N_{\mathrm{e}} s$, determines fixation probability. $N_{\mathrm{e}}$ is raised by subdivision, but $s_{\mathrm{e}}$ is lowered such that $N_{\mathrm{e}} s_{\mathrm{e}}$ is unaltered by subdivision. This framework is consistent with the fact that although fixation probabilities are unaffected by subdivision, times to fixation are increased.

If the assumption of simple genic selection is relaxed-if there is dominance for fitness or frequencydependent selection-fixation probabilities are altered by subdivision, even in the absence of extinction and recolonization (Slatkin 1981; Lande 1985; Spirito et al. 1993; Cherry 2003a). Specifically, subdivision decreases the importance of dominance or frequency dependence. This effect can be described in terms of effective values of the additional parameters that describe the more complex selection regime (CHERry 2003a).

Extinction and recolonization can alter fixation probabilities, even with simple genic selection (BARTON 1993). The effect of this type of population structure is to decrease the importance of selection relative to stochastic forces; extinction and recolonization is an additional stochastic force that brings with it no additional directional change. The interaction between natural selection and extinction and recolonization has been a subject of much recent theoretical work (CHERRY 2003b; Roze and Rousset 2003; Whitlock 2003).

Cherry (2003b) derived results for genic selection in a finite island model with extinction and recoloniza- 
tion by a single founding allele. Here I extend these results in two ways. First, I allow for recolonization by multiple founders, which may have a tendency to originate from the same deme. Second, I obtain results that apply when there is dominance for fitness or, what is formally equivalent, a form of frequency-dependent selection.

\section{MODELS AND RESULTS}

The model of population structure considered here is the finite island model. In this model $D$ demes, each consisting of $N$ haploid or $N / 2$ diploid individuals, exchange migrants among themselves and also serve as sources for recolonization of extinct demes. The expected fraction of migrant alleles entering a deme in a generation is $m$. The probability of extinction of any given deme in any generation is $\lambda$. Subsequent to extinction, a deme is recolonized by $k$ founding alleles. The subpopulation then immediately grows to full size.

Every deme receives migrants and colonists from the population as a whole, in which the allele frequency is $\bar{x}$. Suppose that $\bar{x}$ changes slowly compared to the allele frequency in any deme. From the point of view of any deme, the population as a whole looks, in the short term, much like a source population with constant allele frequency $\bar{x}$. The distribution of the within-deme allele frequencies will then attain a quasi-equilibrium, which corresponds to the equilibrium of a source-sink (continent-island) model. For $\bar{x}$ to change sufficiently slowly, two conditions must hold. First, the number of demes must be large, so that the stochastic change in the overall allele frequency is small compared to the within-deme stochastic change. Second, selection must be weak in the sense that the magnitudes of selective differences are small compared to the reciprocal of the deme size $N$. This condition allows selection to have a significant effect on the behavior of an allele in the population as a whole because the size of the entire population is much larger than $N$. This assumption has the additional consequence that selection can be neglected in the derivation of the quasi-equilibrium distribution of withindeme allele frequency. I make these two assumptionsthat the number of demes is large and that selection is weak compared to $1 / \mathrm{N}$-in all that follows.

The temporal trajectory of an allele's frequency is the outcome of the interaction between the directional effects of natural selection and the stochastic effects of genetic drift and extinction/recolonization. A diffusion approximation for these processes gives a complete statistical description of this trajectory. The diffusion is specified completely by expressions for the mean and variance of the per-generation change in allele frequency as functions of the allele frequency. For the cases considered here, the diffusion turns out to be approximately the same as that describing a panmictic population whose size and selection parameters are different from those of the subdivided population. The parameters of this equivalent panmictic population are, by definition, the effective population size $\left(N_{\mathrm{e}}\right)$, the effective selection coefficient $\left(s_{\mathrm{e}}\right)$, and, in the case of dominance, the effective dominance parameter $\left(h_{\mathrm{e}}\right)$.

Founders chosen independently: I first consider the case of genic selection where the founders that recolonize an extinct deme have no particular tendency to originate from a common source deme. This case corresponds to SLATKIN's (1977) “migrant pool” model.

The mean change in allele frequency in the population as a whole is the mean of the within-deme mean changes due to selection (because migration and recolonization are symmetric they do not, on average, change the allele frequency). For a deme whose allele frequency is $x$, the mean change due to selection is approximately $s x(1-x)$, where $s$ is the selection coefficient. Thus the expected value of the mean change in the entire population is $\sim s E[x(1-x)]$, where the expectation is taken across the quasi-equilibrium distribution of $x$. The quasi-equilibrium value of $E[x(1-x)]$ can be obtained from a recursion that gives this expected value in one generation as a function of its value in the previous generation and the previous value of $E[x]$. Let $H=$ $E[2 x(1-x)]$. This quantity is the probability that two copies of the gene sampled from the same deme, independently and with replacement, are in distinct allelic states. Let $H_{t}$ be the value of $H$ in generation $t$. We seek an expression for $H_{t+1}$ in terms or $H_{t}$ and $E[x]$.

For two copies of the gene sampled at time $t+1$ to be in different allelic states, it is necessary that the same copy of the locus has not been sampled twice (probability $1-1 / N)$. Assuming that this is the case there are several possibilities to consider. There may have just been an extinction/recolonization event (probability $\lambda)$, in which case the two copies of the gene may or may not be descended from distinct founders (probabilities $1-1 / k$ and $1 / k$, respectively). The two can be allelically distinct only if they are descended from distinct founders, in which case they are different with probability $2 \bar{x}(1-\bar{x})$. If there has not just been an extinction/recolonization event (probability $1-\lambda$ ), zero, one, or both of the sampled alleles may be migrants. If neither is a migrant, the immediate ancestors of the sampled pair are chosen independently and with replacement from generation $t$. Thus the probability that they are allelically distinct is $H_{t}$. If exactly one is a migrant, the probability is $E[x](1-\bar{x})+(1-E[x]) \bar{x}$, where $E[x]$ refers to the expectation in the previous generation. If both are migrants, they are chosen independently from the population at large, so the probability that they are in different allelic states is $2 \bar{x}(1-\bar{x})$. Therefore

$$
\begin{aligned}
& H_{t+1}=(1-1 / N) \\
& \times\left\{( 1 - \lambda ) \left[(1-m)^{2} H_{t}+2 m(1-m)(E[x](1-\bar{x})+(1-E[x]) \bar{x})\right.\right. \\
& \left.+m^{2} 2 \bar{x}(1-\bar{x})\right] \\
& +\lambda(1-1 / k) \bar{x}(1-\bar{x})\},
\end{aligned}
$$


where again $E[x]$ refers to the expectation in the previous generation. At equilibrium, $E[x]=\bar{x}$. The equilibrium condition for $H$ is therefore

$$
\begin{gathered}
H=(1-1 / N)\left\{(1-\lambda)\left[(1-m)^{2} H+\left(2 m-m^{2}\right) 2 \bar{x}(1-\bar{x})\right]\right. \\
+\lambda(1-1 / k) 2 \bar{x}(1-\bar{x})\} .
\end{gathered}
$$

Solution for $H$ gives

$$
\begin{aligned}
H & =\frac{(1-1 / N)\left[(1-\lambda)\left(2 m-m^{2}\right)+\lambda(1-1 / k)\right]}{1-(1-1 / N)(1-\lambda)(1-m)^{2}} 2 \bar{x}(1-\bar{x}) \\
& =\frac{(N-1)\left[(1-\lambda)\left(2 m-m^{2}\right)+\lambda(1-1 / k)\right]}{N-(N-1)(1-\lambda)(1-m)^{2}} 2 \bar{x}(1-\bar{x}) .
\end{aligned}
$$

$F_{\mathrm{ST}}$ is defined by $1-F_{\mathrm{ST}} \equiv E[x(1-x)] / \bar{x}(1-\bar{x})=$ $H / 2 \bar{x}(1-\bar{x})$. Because $H$ is proportional to $\bar{x}(1-\bar{x})$, $F_{\text {ST }}$ will be independent of $\bar{x}$, as it is in the absence of extinction and recolonization. From Equation 3 it follows that

$$
\begin{aligned}
1-F_{\mathrm{ST}} & =\frac{(N-1)\left[(1-\lambda)\left(2 m-m^{2}\right)+\lambda(1-1 / k)\right]}{N-(N-1)(1-\lambda)(1-m)^{2}} \\
& \approx \frac{2 N m+N \lambda(1-1 / k)}{2 N m+N \lambda+1}
\end{aligned}
$$

where the approximate equality holds for small $m$, large $N$, and small $\lambda$. For $\lambda=0$, the approximate expression reduces to $2 \mathrm{Nm} /(2 \mathrm{Nm}+1)$, a familiar approximation for $1-F_{\mathrm{ST}}$ for an island model with ordinary migration (Wright 1940; Dobzhansky and Wright 1941).

The above shows that the mean change in $\bar{x}$ in a generation, $M_{\Delta \bar{x}}$, is approximated by $\left(1-F_{\mathrm{ST}}\right) s \bar{x}(1-$ $\bar{x}$ ), with $F_{\mathrm{ST}}$ given by Equation 4 . Because $F_{\mathrm{ST}}$ is independent of $\bar{x}$, the effective selection coefficient can be defined by $M_{\Delta \bar{x}}=s_{\mathrm{e}} \bar{x}(1-\bar{x}) . s_{\mathrm{e}}$ is equal to $\left(1-F_{\mathrm{ST}}\right) s$, and therefore

$$
\begin{aligned}
s_{\mathrm{e}} & =\left(\frac{(N-1)\left[(1-\lambda)\left(2 m-m^{2}\right)+\lambda(1-1 / k)\right]}{N-(N-1)(1-\lambda)(1-m)^{2}}\right) s \\
& \approx\left(\frac{2 N m+N \lambda(1-1 / k)}{2 N m+N \lambda+1}\right) s .
\end{aligned}
$$

The effective population size can be defined in terms of the variance in the change in overall allele frequency in a generation $(\Delta \bar{x})$. This overall variance is a combination of within-deme variances. The variance of the change within a deme is a function of the local allele frequency. To obtain the variance in $\Delta \bar{x}, V_{\Delta \bar{x}}$, I first derive an expression for the within-deme variance conditional on allele frequency. I then take the mean across the quasi-equilibrium distribution of allele frequency, which leads to an approximate expression for the overall variance.

Let $x$ be the allele frequency within a representative deme in one generation and $x^{\prime}$ be the frequency in the next. The variance of $\Delta x$, the change in allele frequency, is equal to the variance of $x^{\prime}$, which is the second moment of $x^{\prime}$ about $E\left[x^{\prime}\right]$. Because the mean change in allele frequency in a single generation is small for $s, m$, $\lambda \ll 1, E\left[x^{\prime}\right] \approx x$. The distribution of $x^{\prime}$ is a mixture of two components, one corresponding to extinction and recolonization and another to the binomial sampling that occurs in the absence of extinction. Conditional on no extinction, the second moment about $E\left[x^{\prime}\right]$ is $\sim(1 / N) x(1-x)$ (the familiar binomial sampling variance). The expected value of this quantity over the distribution of $x$ is $(1 / N)\left(1-F_{\mathrm{ST}}\right) \bar{x}(1-\bar{x})$. The case of extinction and recolonization is more complicated. Let $y$ be the allele frequency after recolonization. The variance of $y$ is about equal to the variance in the allele frequency among the founders. The number of the $k$ founders that carry the $A$ allele has a binomial distribution with number of "trials" $k$ and probability of "success" $\bar{x}$. The variance in the number of $A$ alleles is $k \bar{x}(1-\bar{x})$, and the variance in the fraction of $A$ alleles is $(1 / k) \bar{x}(1-\bar{x})$. Thus, since the mean of $y$ is $\bar{x}$, the second moment of $y$ about zero, $E\left[y^{2}\right]$, is $\sim(1 / k) \bar{x}(1-$ $\bar{x})+\bar{x}^{2}$. The second moment about $x, E\left[(y-x)^{2}\right]=$ $E\left[y^{2}\right]-2 E[y] x+x^{2}$, is $\sim(1 / k) \bar{x}(1-\bar{x})+\bar{x}^{2}-2 \bar{x} x+$ $x^{2}$. We know that at equilibrium $E[x]=\bar{x}$ and $E[x(1-$ $x)]=\left(1-F_{\mathrm{ST}}\right) \bar{x}(1-\bar{x})$, and therefore $E\left[x^{2}\right]=\bar{x}-$ $\left(1-F_{\mathrm{ST}}\right) \bar{x}(1-\bar{x})$. It follows from substitution and rearrangement that the expected value of the second moment of $y$ about $x$ is $\sim\left[(1 / k)+F_{\mathrm{ST}}\right] \bar{x}(1-\bar{x})$. Combining the two components of the variance of $x^{\prime}$ gives

$$
E\left[\operatorname{Var}\left(x^{\prime}\right)\right] \approx\left\{(1-\lambda) \frac{1}{N}\left(1-F_{\mathrm{ST}}\right)+\lambda\left[\frac{1}{k}+F_{\mathrm{ST}}\right]\right\} \bar{x}(1-\bar{x}) .
$$

This is the expected variance of the change in a single deme. The variance of $\Delta \bar{x}$, the change in the overall allele frequency, is given by

$$
\begin{aligned}
V_{\Delta \bar{x}} & =\frac{1}{D^{2}} \sum_{i} \operatorname{Var}\left(\Delta x_{i}\right) \\
& \approx \frac{1}{D} E\left[\operatorname{Var}\left(\Delta x_{i}\right)\right] \\
& \approx \frac{1}{D}\left\{(1-\lambda) \frac{1}{N}\left(1-F_{\mathrm{ST}}\right)+\lambda\left[\frac{1}{k}+F_{\mathrm{ST}}\right]\right\} \bar{x}(1-\bar{x}),
\end{aligned}
$$

where $x_{i}$ is the allele frequency in the $i$ th deme. Thus $V_{\Delta \bar{x}}$ is approximately proportional to $\bar{x}(1-\bar{x})$, as it is in a panmictic population, and the variance effective size can be defined by $V_{\Delta \bar{x}}=\left(1 / N_{\mathrm{e}}\right) \bar{x}(1-\bar{x})$. From this definition and Equation 8 it follows that

$$
N_{\mathrm{e}} \approx \frac{N D}{\left(1-F_{\mathrm{ST}}\right)+N \lambda\left[(1 / k)+F_{\mathrm{ST}}\right]},
$$

with $F_{\mathrm{ST}}$ given by Equation $4 . N_{\mathrm{e}}$ can be larger or smaller than the actual population size $N D$, depending on the parameters $N, m, \lambda$, and $k$.

Founders that tend to come from the same deme: So far it has been assumed that the individuals that recolonize an extinct deme have no particular tendency to originate from a common deme. It is biologically plausible that founders do tend to come from the same 
deme. Slatkin (1977) analyzed a model (the "propagule pool" model) in which all of the founders always come from the same deme. Whitlock and Barton (1997) presented more general results that allowed for any degree of the tendency for a common deme of origin. The methods used above can be adapted to give results for alleles under selection with this pattern of recolonization.

Following Whitlock and Barton (1997), but using a haploid model, let $\phi$ be the probability that two distinct founders come from the same deme. As above, we seek an equilibrium value of $H$, the probability that two genes sampled from within a deme are allelically distinct. Now there are two cases to consider when a sampled pair are immediate descendants of distinct founders. With probability $1-\phi$ the founders come from different demes and the conditional probability that they carry distinct alleles is $2 \bar{x}(1-\bar{x})$. With probability $\phi$ they come from the same deme and the conditional probability that they are distinct is $H$ (of the previous generation). The equilibrium condition for $H$ is therefore

$$
\begin{array}{r}
H=(1-1 / N)\left\{(1-\lambda)\left[(1-m)^{2} H+\left(2 m-m^{2}\right) 2 \bar{x}(1-\bar{x})\right]\right. \\
+\lambda(1-1 / k)[(1-\phi) 2 \bar{x}(1-\bar{x})+\phi H]\}
\end{array}
$$

(compare to Equation 2). Solving for $H$, and using $1-$ $F_{\mathrm{ST}}=H / 2 \bar{x}(1-\bar{x})$, we obtain

$$
\begin{aligned}
1-F_{\mathrm{ST}} & =\frac{(1-1 / N)\left[(1-\lambda)\left(2 m(1-m)+m^{2}\right)+\lambda(1-1 / k)(1-\phi)\right]}{1-(1-1 / N)(1-\lambda)(1-m)^{2}-(1-1 / N) \lambda(1-1 / k) \phi} \\
& \approx \frac{2 N m+N \lambda(1-1 / k)(1-\phi)}{2 N m+N \lambda[1-\phi(1-1 / k)]+1} .
\end{aligned}
$$

The approximate equality is equivalent to an expression for $F_{\text {ST }}$ given by WHITLOCK and BARTON (1997, Equation 21).

Again $F_{\mathrm{ST}}$ is independent of $\bar{x}$, so $s_{\mathrm{e}}$ is well defined. It is given by

$$
\begin{aligned}
s_{\mathrm{e}} & =\left(\frac{(1-1 / N)\left[(1-\lambda)\left(2 m(1-m)+m^{2}\right)+\lambda(1-1 / k)(1-\phi)\right]}{1-(1-1 / N)(1-\lambda)(1-m)^{2}-(1-1 / N) \lambda(1-1 / k) \phi}\right) s \\
& \approx\left(\frac{2 N m+N \lambda(1-1 / k)(1-\phi)}{2 N m+N \lambda[1-\phi(1-1 / k)]+1}\right) s .
\end{aligned}
$$

I now turn to the variance of the change in allele frequency in a deme, which leads to an expression for the effective population size. The component of the variance due to ordinary drift is the same as in Equation 7 , but the component due to extinction and recolonization must be modified. The number of $A$ alleles among the $k$ founders is the sum of $k$ Bernoulli random variables, $Y_{i}$, that are 1 when the $i$ th founder carries an $A$ allele (probability $\bar{x}$ ) and 0 when it carries an $a$ (probability $1-\bar{x}$ ). When founders are chosen without regard to the deme of origin, these Bernoulli random variables are independent, and the distribution is a binomial. In the more general case the $Y_{i}$ are not independent and we must consider their covariance to compute the vari- ance of their sum. The covariance of a pair is given by $\operatorname{Cov}\left(\Upsilon_{i}, \Upsilon_{j}\right)=E\left[\Upsilon_{i} \Upsilon_{j}\right]-E\left[\Upsilon_{i}\right] E\left[\Upsilon_{j}\right]$. Clearly $E\left[\Upsilon_{i}\right] E\left[\Upsilon_{j}\right]=$ $\bar{x}^{2} . E\left[Y_{i} Y_{j}\right]$ is the probability that both the $i$ th and $j$ th founders carry the $A$ allele. Consider the case where $i \neq$ $j$. With probability $1-\phi$, the corresponding founders come from different demes and the conditional probability that both carry $A$ is $\bar{x}^{2}$. With probability $\phi$ they come from the same deme, and the conditional probability that they both carry the $A$ allele is $E\left[x_{i}^{2}\right]=E\left[x_{i}\right]-$ $E\left[x_{i}\left(1-x_{i}\right)\right]=\bar{x}-\left(1-F_{\mathrm{ST}}\right) \bar{x}(1-\bar{x})=F_{\mathrm{ST}} \bar{x}(1-\bar{x})+$ $\bar{x}^{2}$. Therefore the unconditional probability that both carry $A$ is $(1-\phi) \bar{x}^{2}+\phi\left[F_{\mathrm{ST}} \bar{x}(1-\bar{x})+\bar{x}^{2}\right]=\phi F_{\mathrm{ST}} \bar{x}(1-$ $\bar{x})+\bar{x}^{2}$ and

$$
\operatorname{Cov}\left(Y_{i}, Y_{j}\right)=\phi F_{\mathrm{ST}} \bar{x}(1-\bar{x})
$$

The variance of $Y_{i}$ is simply the Bernoulli variance $\bar{x}(1-$ $\bar{x})$. The variance of the sum of the $Y_{i}$ is the sum of their variances plus twice the sum of the covariances of distinct pairs:

$$
\begin{aligned}
\operatorname{Var}\left(\sum \Upsilon_{i}\right) & =k \operatorname{Var}\left(\Upsilon_{i}\right)+k(k-1) \operatorname{Cov}\left(\Upsilon_{i}, \Upsilon_{j}\right) \\
& =\left[k+k(k-1) \phi F_{\mathrm{ST}}\right] \bar{x}(1-\bar{x}) .
\end{aligned}
$$

The variance of the fraction of $A$ alleles in the newly formed population is given approximately by

$$
\operatorname{Var}(y) \approx\left[\frac{1}{k}+\frac{k-1}{k} \phi F_{\mathrm{ST}}\right] \bar{x}(1-\bar{x}) .
$$

Thus the analog of Equation 7 for the more general case is

$E\left[\operatorname{Var}\left(x^{\prime}\right)\right] \approx\left\{(1-\lambda) \frac{1}{N}\left(1-F_{\mathrm{ST}}\right)+\lambda\left[\frac{1}{k}+\left(\frac{k-1}{k} \phi+1\right) F_{\mathrm{ST}}\right]\right\} \bar{x}(1-\bar{x})$.

This leads to

$$
V_{\Delta \bar{x}} \approx \frac{1}{D}\left\{(1-\lambda) \frac{1}{N}\left(1-F_{\mathrm{ST}}\right)+\lambda\left[\frac{1}{k}+\left(\frac{k-1}{k} \phi+1\right) F_{\mathrm{ST}}\right]\right] \bar{x}(1-\bar{x})
$$

and

$N_{\mathrm{e}} \approx \frac{N D}{\left(1-F_{\mathrm{ST}}\right)+N \lambda\left[(1 / k)+(((k-1) / k) \phi+1) F_{\mathrm{ST}}\right]}$

(compare to Equations 8 and 9). Equation 19 can be shown, with the aid of Equation 11, to be equivalent to a result obtained by WHITLOCK and BARTON (1997).

These results show that a selected allele in a subdivided population with extinction and recolonization behaves much like an allele with a different selection coefficient in a panmictic population with a different size. This follows from the fact that both the mean and the variance of the change in allele frequency are approximately proportional to $\bar{x}(1-\bar{x})$, as they are in a panmictic population (expressions for this mean and variance completely determine the diffusion approximation). The parameters of the equivalent panmictic population, 
$s_{\mathrm{e}}$ and $N_{\mathrm{e}}$, are given by Equations 12 and 19. The value of $N_{\mathrm{e}} s_{\mathrm{e}}$, which determines fixation probability, is altered by subdivision with extinction and recolonization. Specifically,

$$
N_{\mathrm{e}} s_{\mathrm{e}} \approx \frac{\left(1-F_{\mathrm{ST}}\right)}{\left(1-F_{\mathrm{ST}}\right)+N \lambda\left[(1 / k)+(((k-1) / k) \phi+1) F_{\mathrm{ST}}\right]} N D s
$$

and the effect of extinction and recolonization is to reduce the magnitude of $N_{e} s_{e}$.

More complicated patterns of colonization: In the foregoing it was assumed that the number of founders was fixed and that they contributed equally to the new population. The number of founders might vary randomly, and the contributions of founders might be unequal. For the present purposes, these details can be summed up by a single number $\alpha$, the probability that two distinct copies of the gene in a newly recolonized deme descend from the same founding allele. Suppose, for example, that the number of founders is one with probability $1 / 4$, two with probability $1 / 2$, and three with probability $1 / 4$. Then $\alpha=(1 / 4)(1)+(1 / 2)(1 / 2)+$ $(1 / 4)(1 / 3)=7 / 12$. More generally, $\alpha$ equals the expected value of $1 / k$ when founders make equal contributions to the new population. Suppose, to take another example, that there are always two founders, but that one gives rise to, on average, a fraction $a$ of the new population while the other gives rise to the remaining $1-a$. Then $\alpha=a^{2}+(1-a)^{2}=1-2 a(1-a)$. Because $\alpha=1 / k$ with fixed $k$ and equal contributions, it makes sense to define the effective number of founders, $\hat{k}$, by $\alpha=1 / \hat{k}$.

Expressions for $F_{\mathrm{ST}}$, and hence $s_{\mathrm{e}}$, for the present generalization are straightforward modifications of results for the special case presented earlier. In Equation 10, the number of founders $k$ matters only in that it determines the probability of descent from the same founder. Thus simply replacing $k$ with $\hat{k}$ gives the more general result:

$$
\begin{aligned}
1-F_{\mathrm{ST}} & =\frac{(1-1 / N)\left[(1-\lambda)\left(2 m(1-m)+m^{2}\right)+\lambda(1-1 / \hat{k})(1-\phi)\right]}{1-(1-1 / N)(1-\lambda)(1-m)^{2}-(1-1 / N) \lambda(1-1 / \hat{k}) \phi} \\
& \approx \frac{2 N m+N \lambda(1-1 / \hat{k})(1-\phi)}{2 N m+N \lambda[1-\phi(1-1 / \hat{k})]+1} .
\end{aligned}
$$

As before, $s_{\mathrm{e}}=\left(1-F_{\mathrm{ST}}\right) s$.

That $\alpha=1 / \hat{k}$ is sufficient to determine the variance of $\Delta x$ is more difficult to show. The number of $A$ alleles in a deme after recolonization is the sum of $N$ Bernoulli random variables, $v_{i}$, that are 1 if the $i$ th individual carries an $A$ allele and 0 otherwise. The variance of $v_{i}$ is $\bar{x}(1-\bar{x})$. We seek the covariance of $v_{i}$ and $v_{j}$ for $i \neq$ $j$. This is given by $\operatorname{Cov}\left(v_{i}, v_{j}\right)=E\left[v_{i} v_{j}\right]-E\left[v_{i}\right] E\left[v_{j}\right] . E\left[v_{i}\right]=$ $\bar{x}$, so $E\left[v_{i}\right] E\left[v_{j}\right]=\bar{x}^{2} . E\left[v_{i} v_{j}\right]$ is the probability that two distinct copies of the gene are both $A$ 's. This is equal to $(1 / \hat{k}) \bar{x}+(1-1 / \hat{k})\left[(1-\phi) \bar{x}^{2}+\phi\left(F_{\mathrm{ST}} \bar{x}(1-\bar{x})+\bar{x}^{2}\right)\right]=$ $(1 / \hat{k}) \bar{x}(1-\bar{x})+(1-1 / \hat{k}) \phi F_{\mathrm{ST}} \bar{x}(1-\bar{x})+\bar{x}^{2}$. Thus

$$
\operatorname{Cov}\left(v_{i}, v_{j}\right)=\left[1 / \hat{k}+(1-1 / \hat{k}) \phi F_{\mathrm{ST}}\right] \bar{x}(1-\bar{x}) .
$$

The variance in the sum of the $v_{i}$ is given by

$$
\begin{aligned}
\operatorname{Var}\left(\sum\left(v_{i}\right)\right) & =N \operatorname{Var}\left(v_{i}\right)+N(N-1) \operatorname{Cov}\left(v_{i}, v_{j}\right) \\
& =N \bar{x}(1-\bar{x})+N(N-1)\left[1 / \hat{k}+(1-1 / \hat{k}) \phi F_{\text {ST }}\right] \bar{x}(1-\bar{x})
\end{aligned}
$$

and the variance in the allele frequency after recolonization is given by

$$
\operatorname{Var}(y)=\left[\frac{N-1}{N}\left(\frac{1}{\hat{k}}+\frac{\hat{k}-1}{\hat{k}} \phi F_{\mathrm{ST}}\right)+\frac{1}{N}\right] \bar{x}(1-\bar{x}) .
$$

For $\hat{k}=k$, this equation differs slightly from Equation 16. The reason is that Equation 16 took the variance in allele frequency in the new population to be equal to the variance among the founders, whereas Equation 24 includes additional variance due to multinomial sampling of the founders in the formation of the new population. Which expression is more appropriate depends on the details of the colonization process, but the effect of the difference on $N_{\mathrm{e}}$ is negligible. This is evident from comparison of Equation 19 with the expression for $N_{\mathrm{e}}$ corresponding to Equation 24:

$N_{\mathrm{e}} \approx \frac{N D}{\left(1-F_{\mathrm{ST}}\right)+(N-1) \lambda\left[(1 / \hat{k})+(((\hat{k}-1) / \hat{k}) \phi+1) F_{\mathrm{ST}}\right]+\lambda}$.

Dominance or local frequency dependence: Suppose that in a diploid population the fitnesses of the genotypes $a a, A a$, and $A A$ are given by $1,1+2 h s$, and $1+$ $2 s$, respectively. The average selective difference between an $A$ and an $a$ allele, the marginal selection coefficient $\hat{s}$, depends on the allele frequency $x$ and is given by $\hat{s}(x)=2 h s(1-x)+(2 s-2 h s) x$ in an unstructured population with nonassortative mating. $\hat{s}$ can be written as $k_{0}+k_{1} x$, with $k_{0}=2 h s$ and $k_{1}=2 s-4 h s$. Thus the effect of dominance on fitness is formally equivalent to frequency-dependent selection where fitness is a linear function of allele frequency.

Consider now a subdivided population. The expected change in overall allele frequency $\bar{x}$ is the mean of the expected changes due to selection across all demes:

$$
\begin{aligned}
E[\Delta \bar{x}] & =(1 / D) \sum_{i} \hat{s}\left(x_{i}\right) x_{i}\left(1-x_{i}\right) \\
& \approx k_{0} E\left[x_{i}\left(1-x_{i}\right)\right]+k_{1} E\left[x_{i}^{2}\left(1-x_{i}\right)\right] .
\end{aligned}
$$

We have already established that $E\left[x_{i}\left(1-x_{i}\right)\right]=(1-$ $\left.F_{\mathrm{ST}}\right) \bar{x}(1-\bar{x})$ at quasi-equilibrium, with $F_{\mathrm{ST}}$ given by Equation 4. The problem is then to find $E\left[x_{i}^{2}\left(1-x_{i}\right)\right]$. In the absence of extinction and recolonization, this expectation followed from the moments of the beta distribution that approximately characterizes within-deme allele frequency under that condition (WHITLOCK 2002; Cherry 2003a). In the presence of extinction and recolonization this distribution is unknown. However, just as we derived quasi-equilibrium conditions for $H=$ $2 E\left[x_{i}\left(1-x_{i}\right)\right]$ (Equations 2 and 10), we can write such 
conditions for $E\left[x_{i}^{2}\left(1-x_{i}\right)\right]$. As in the case of $E\left[x_{i}(1-\right.$ $\left.x_{i}\right)$ ], it is convenient to think in terms of probabilities of sampling certain allele configurations. Let $I=$ $E\left[3 x_{i}^{2}\left(1-x_{i}\right)\right] . I$ is then the probability that three copies of the gene sampled from the same deme, independently and with replacement, consist of two copies of the $A$ allele and one of the $a$ allele. Consideration of the ways in which such a sample can arise, and of their probabilities as functions of conditions in the previous generation, yields the desired quasi-equilibrium condition for $I$.

To limit the number of cases that must be considered, I immediately adopt approximations for small $m$ and $\lambda$ and large $N$ that neglect possibilities involving two or more unlikely events. For example, the probability that no member of a triple is a migrant is approximated by $1-3 m$, the probability that exactly one is a migrant is approximated by $3 \mathrm{~m}$, and the possibility that more than one is a migrant is neglected. Even with such simplifications, there are many cases to consider, which I enumerate hierarchically below for the migrant pool model.

1. With probability $1-3 / N$, all three members of the sample are distinct (no copy of the locus has been sampled twice).

a. With probability $1-\lambda$, extinction has not occurred.

i. With probability $1-3 m$, none of the sampled alleles is a migrant. In this case the probability of two $A$ 's and one $a$ is the value of $I$ in the previous generation.

ii. With probability $3 \mathrm{~m}$, one member of the sample is a migrant. There are two ways that there can be two $A$ 's and one $a$. The two nonmigrants can be allelically distinct and the migrant a copy of the $A$ allele [probability $H \bar{x}=2(1-$ $\left.\left.F_{\mathrm{ST}}\right) \bar{x}^{2}(1-\bar{x})\right]$, or both nonmigrants can be $A$ 's and the migrant an $a$ [probability $E\left[x^{2}\right](1-$ $\bar{x})=E[x-x(1-x)](1-\bar{x})=F_{\mathrm{ST}} \bar{x}(1-\bar{x})+$ $\left.\left(1-F_{\mathrm{ST}}\right) \bar{x}^{2}(1-\bar{x})\right]$. The total probability is therefore $F_{\mathrm{ST}} \bar{x}(1-\bar{x})+3\left(1-F_{\mathrm{ST}}\right) \bar{x}^{2}(1-\bar{x})$.

b. With probability $\lambda$ an extinction/recolonization event has occurred.

i. With probability $(1-1 / k)(1-2 / k)$ the three members of the sample are descendants of distinct founders, and the probability of two $A$ 's and one $a$ is $3 \bar{x}^{2}(1-\bar{x})$.

ii. With probability $3(1 / k)(1-1 / k)$ two of the sampled alleles are descendants of the same founder and the third is descended from another founder. There will be two A's and one $a$ when the first founder carries an $A$ and the second an $a$, which happens with probability $\bar{x}(1-\bar{x})$.

iii. With probability $1 / k^{2}$ all three members of the sample descend from the same founder and two $A$ 's and one $a$ are impossible.
2. With probability $3 / N$, one pair of the triple represents resampling of the same copy of the locus. There will be two $A$ 's and one $a$ when the twice-sampled copy is an $A$ and the other is an $a$, which happens with probability $H / 2$.

Putting this all together gives

$$
\begin{gathered}
I \approx(1-3 / N)\left\{( 1 - \lambda ) \left[(1-3 m) I+3 m\left(3\left(1-F_{\mathrm{ST}}\right) \bar{x}^{2}(1-\bar{x})\right.\right.\right. \\
\left.\left.+F_{\mathrm{ST}} \bar{x}(1-\bar{x})\right)\right] \\
+\lambda\left[3(1-1 / k)(1-2 / k) \bar{x}^{2}(1-\bar{x})\right. \\
+3(1 / k)(1-1 / k) \bar{x}(1-\bar{x})]\} \\
+(3 / N)\left(1-F_{\mathrm{ST}}\right) \bar{x}(1-\bar{x})
\end{gathered}
$$

at quasi-equilibrium. Solution for $I$ gives

$$
\begin{gathered}
I \approx\left[( 1 - 3 / N ) \left\{3 m\left[3\left(1-F_{\mathrm{ST}}\right) \bar{x}^{2}(1-\bar{x})+F_{\mathrm{ST}} \bar{x}(1-\bar{x})\right]\right.\right. \\
+\lambda\left[3(1-1 / k)(1-2 / k) \bar{x}^{2}(1-\bar{x})\right. \\
+3(1 / k)(1-1 / k) \bar{x}(1-\bar{x})]\} \\
\left.+(3 / N)\left(1-F_{\mathrm{ST}}\right) \bar{x}(1-\bar{x})\right] /[1-(1-3 / N)(1-\lambda)(1-3 m)] .
\end{gathered}
$$

Neglecting second-order terms in the denominator, using $1-3 / N \approx 1$, and multiplying numerator and denominator by $N / 3$ gives

$$
\begin{aligned}
I \approx\left[N m\left[3\left(1-F_{\mathrm{ST}}\right) \bar{x}^{2}(1-\bar{x})+F_{\mathrm{ST}} \bar{x}(1-\bar{x})\right]\right. \\
+(N / 3) \lambda\left[3(1-1 / k)(1-2 / k) \bar{x}^{2}(1-\bar{x})\right. \\
+3(1 / k)(1-1 / k) \bar{x}(1-\bar{x})] \\
\left.+\left(1-F_{\mathrm{ST}}\right) \bar{x}(1-\bar{x})\right] /[N m+N \lambda / 3+1] .
\end{aligned}
$$

This daunting expression can be written in the form $c_{0} \bar{x}(1-\bar{x})+c_{1} \bar{x}^{2}(1-\bar{x})$, with $c_{0}$ and $c_{1}$ independent of $\bar{x}$ and given by

$$
\begin{aligned}
c_{0} & =\frac{N m F_{\mathrm{ST}}+N \lambda(1 / k)(1-1 / k)+\left(1-F_{\mathrm{ST}}\right)}{N m+N \lambda / 3+1} \\
c_{1} & =\frac{3 N m\left(1-F_{\mathrm{ST}}\right)+N \lambda(1-1 / k)(1-2 / k)}{N m+N \lambda / 3+1} .
\end{aligned}
$$

Thus the expected mean change in population-wide allele frequency, $E[\hat{s}(x) x(1-x)]=k_{0} H / 2+k_{1} I / 3$, can be written as $\left(k_{0 \mathrm{e}}+k_{\mathrm{le}} \bar{x}\right) \bar{x}(1-\bar{x})$, as it can in a panmictic population with dominance or linear frequency dependence. The constants $k_{0 \mathrm{e}}$ and $k_{1 \mathrm{e}}$ are given by

$$
\begin{aligned}
k_{0 \mathrm{e}} & =\left(1-F_{\mathrm{ST}}\right) k_{0}+\left(c_{0} / 3\right) k_{1} \\
& =\left(1-F_{\mathrm{ST}}\right) k_{0}+\frac{N m F_{\mathrm{ST}}+N \lambda(1 / k)(1-1 / k)+\left(1-F_{\mathrm{ST}}\right)}{3 N m+N \lambda+3} k_{1}
\end{aligned}
$$

and 


$$
\begin{aligned}
k_{\mathrm{le}} & =\left(c_{1} / 3\right) k_{1} \\
& =\frac{3 N m\left(1-F_{\mathrm{ST}}\right)+N \lambda(1-1 / k)(1-2 / k)}{3 N m+N \lambda+3} k_{1} .
\end{aligned}
$$

$k_{0 \mathrm{e}}$ and $k_{\text {le }}$ can be interpreted as the selection parameters that, in a panmictic population of size $N_{\mathrm{e}}$, give roughly the same behavior as do the selection parameters $k_{0}$ and $k_{1}$ in the subdivided population. The selection coefficient and dominance parameter in the equivalent panmictic population, $s_{\mathrm{e}}$ and $h_{\mathrm{e}}$, are given by $s_{\mathrm{e}}=k_{0 \mathrm{e}}+k_{\mathrm{e}} / 2$ and $h_{\mathrm{e}}=k_{0 \mathrm{e}} /\left(2 k_{0 \mathrm{e}}+k_{\mathrm{e}}\right)$. From Equation 4 it follows that $N m F_{\text {ST }}$, which appears in Equation 32, equals (1/2) [ $(1-$ $\left.\left.F_{\mathrm{ST}}\right)+N \lambda\left(1-F_{\mathrm{ST}}\right)-N \lambda(1-1 / k)\right]$. Some algebra then gives

$$
s_{\mathrm{e}}=\left(1-F_{\mathrm{ST}}\right) s
$$

and

$$
h_{\mathrm{e}}=\frac{3 N m\left(1-F_{\mathrm{ST}}\right)+N \lambda(1-1 / k)(1-2 / k)}{\left(1-F_{\mathrm{ST}}\right)(3 N m+N \lambda+3)}(h-1 / 2)+1 / 2 .
$$

Two noteworthy properties of $s_{\mathrm{e}}$ and $h_{\mathrm{e}}$ that were found to hold in the absence of extinction and recolonization (CHerry 2003a) also hold in its presence. First, $s_{\mathrm{e}}$ is independent of $h$ and is directly proportional to $s$. As in the case with no extinction, the proportionality constant is $1-F_{\mathrm{ST}}$, though the value of $F_{\mathrm{ST}}$ is altered by extinction and recolonization. Second, $h_{\mathrm{e}}$ is independent of $s$, and subdivision decreases the deviation of $h_{\mathrm{e}}$ from $1 / 2$ by a factor that is independent of $h$.

If founders tend to come from the same deme, analysis of the case of dominance or frequency dependence is more complicated. There is an increase in the number of cases that must be considered when there is an extinction/recolonization event. Furthermore, for $k>2$ an additional parameter must be specified to describe the pattern of recolonization; $\phi$ is insufficient to do so.

The need for an additional parameter can be seen as follows. For three distinct founders there are three possibilities with regard to demes of origin: (a) The three founders come from three different demes; (b) two come from one deme and the third from another; and (c) all three come from the same deme. Suppose that $\phi=1 / 3$. This value of $\phi$ can be realized in many different ways. At one extreme, possibility c could occur with probability $1 / 3$, possibility a with probability $2 / 3$, and possibility $\mathrm{b}$ with probability 0 . At the other extreme, possibility b could occur with probability 1 . Thus $\phi$ does not uniquely determine the probabilities of the three outcomes.

Let $\psi$ be the probability that all three distinct founders come from the same deme. Then the probabilities of outcomes a, b, and c are $1-3 \phi+2 \psi, 3(\phi-\psi)$, and $\psi$, respectively. Specifying $\phi$ and $\psi$ uniquely determines the probabilities of all possible outcomes.

To account for common demes of origin, item 1.b in the list above must be modified to read as follows: b. With probability $\lambda$ an extinction/recolonization event has occurred.

i. With probability $(1-1 / k)(1-2 / k)$ the three members of the sample are descendants of distinct founders.

A. With probability $1-3 \phi+2 \psi$ the three founders come from different demes, and the probability of two $A$ 's and one $a$ is $3 \bar{x}^{2}(1-\bar{x})$.

B. With probability $3(\phi-\psi)$ two of the founders come from one deme and the third from another. By an argument similar to that for case 1.a.ii, the probability of two $A$ 's and an $a$ is $F_{\mathrm{ST}} \bar{x}(1-\bar{x})+3\left(1-F_{\mathrm{ST}}\right) \bar{x}^{2}(1-\bar{x})$.

C. With probability $\psi$ the three founders come from a single deme. The probability of two A's and an $a$ is $I$ of the previous generation.

ii. With probability $3(1 / k)(1-1 / k)$ two of the sampled alleles are descendants of the same founder and the third is descended from another founder. There will be two A's and one $a$ when the first founder carries an $A$ and the second an $a$.

A. With probability $1-\phi$ the founders do not come from the same deme, and the probability that the first is an $A$ and the second an $a$ is $\bar{x}(1-\bar{x})$.

B. With probability $\phi$ the founders come from the same deme. The probability of an $A$ and an $a$ is $\left(1-F_{\mathrm{ST}}\right) \bar{x}(1-\bar{x})$.

iii. With probability $1 / k^{2}$ all three members of the sample descend from the same founder and two $A$ 's and one $a$ are impossible.

The modified list of possibilities leads to a generalization of Equation 29. Like Equation 29, this generalization can be written as $c_{0} \bar{x}(1-\bar{x})+c_{1} \bar{x}^{2}(1-\bar{x})$. The constants are given by

$$
\begin{aligned}
c_{0}=\left[N m F_{\mathrm{ST}}+N \lambda(1-1 / k)(1-2 / k)(\phi-\psi) F_{\mathrm{ST}}+N \lambda(1 / k)(1-1 / k)\left(1-F_{\mathrm{ST}} \phi\right)\right. \\
\left.+\left(1-F_{\mathrm{ST}}\right)\right] /[N m+N \lambda / 3+1-N \lambda(1-1 / k)(1-2 / k) \psi]
\end{aligned}
$$

and

$$
\begin{aligned}
c_{1}=\left[3 N m\left(1-F_{\mathrm{ST}}\right)+N \lambda(1-1 / k)(1-2 / k)\right. & \\
& \left.\times\left[(1-3 \phi+2 \psi)+3(\phi-\psi)\left(1-F_{\mathrm{ST}}\right)\right]\right] /[N m+N \lambda / 3+1 \\
& -N \lambda(1-1 / k)(1-2 / k) \psi] .
\end{aligned}
$$

Thus the diffusion approximation for the subdivided population with linear frequency dependence is again identical to that for a panmictic population with linear frequency dependence but different size and selection parameters.

\section{COMPUTER SIMULATIONS}

To test the theoretical results obtained here, I have run computer simulations of the population model and 


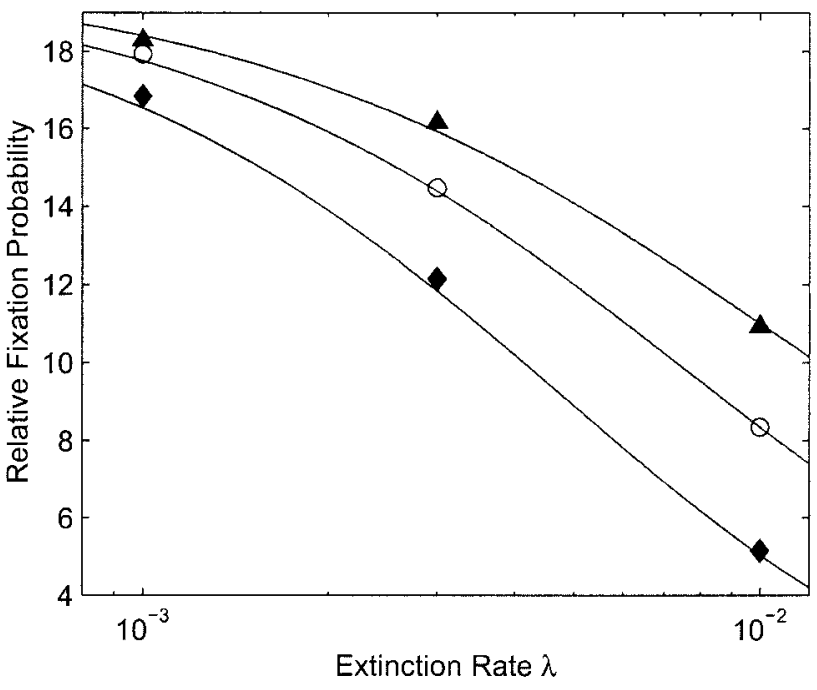

Figure 1.-Predicted and observed fixation probabilities for various values of $k$ and $\lambda$. Fixation probabilities are given relative to that of a neutral allele. In all cases the $A$ allele was initially present in a single copy, $N=100, D=100, m=0.01$, and $s=10^{-3}$. Simulation results and predictions as functions of $\lambda$ are shown for three values of $k: k=1$ (solid diamonds and curve), $k=2$ (open circles and curve), and $k=4$ (solid triangles and curve).

compared the results to analytic predictions. In these simulations the state of the population is represented by $D$ integers, each giving the number of copies of the $A$ allele in a particular deme. Each generation new values of these integers are chosen stochastically in accordance with the model. With probability $\lambda$ an extinction/ recolonization event occurs in a deme. The allele frequency among the founders is chosen probabilistically according to the colonization pattern being simulated, and the number of copies of $A$ is drawn from a binomial distribution with this as its mean. With probability $1-$ $\lambda$ there is no extinction. In this case the new number of $A$ 's is drawn from a binomial whose mean depends on the within-deme frequency in the previous generation, the pattern of selection, the overall allele frequency $\bar{x}$, and the migration rate. Theoretical predictions of fixation probabilities and fixation times result from substitution of $N_{\mathrm{e}}, s_{\mathrm{e}}$, and $h_{\mathrm{e}}$ into classical results for panmictic populations (KImUra 1957; KImURA and Онта 1969).

The effect of the number of founders: These simulations examine the effects of altering the number of founders. The case where there is no tendency for a common deme of origin was simulated. In the case of extinction the number of $A$ 's among the $k$ founders was chosen from a binomial distribution with the probability parameter equal to $\bar{x}$. In the absence of extinction, the number of $A$ alleles in the next generation was chosen from a binomial distribution whose probability parameter $p$ is determined as follows. Let $\tilde{p}=(1-m) x_{i}+$ $m \bar{x}$, where $x_{i}$ is the allele frequency in the deme in

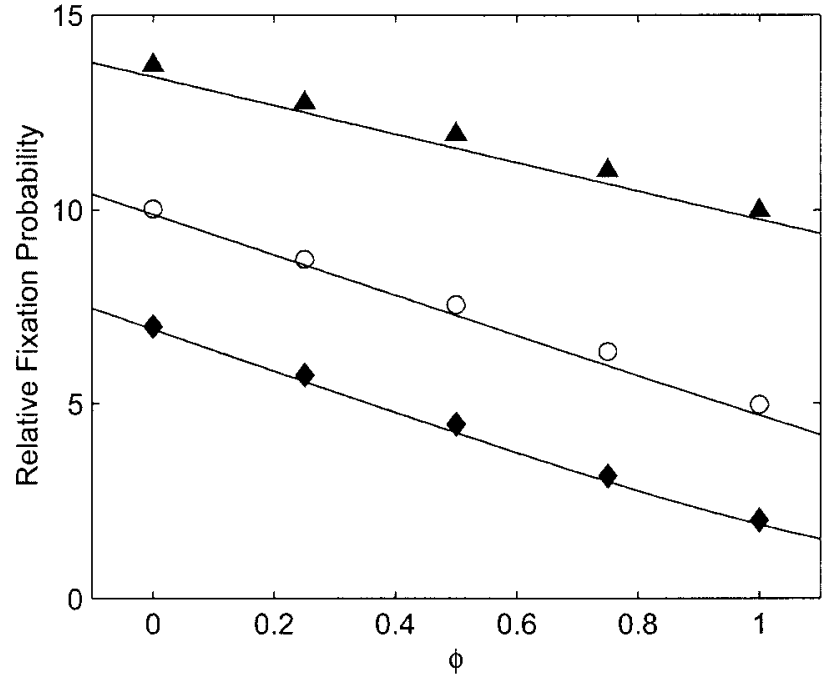

Figure 2.-Predicted and observed relative fixation probabilities when founders have some tendency to come from the same deme. In all cases there are four founders $(k=4), N=$ $100, D=100, m=0.001, s=10^{-3}$, and the $A$ allele was initially present in a single copy. Simulation results and predictions as functions of $\phi$, the probability of a common deme of origin, are shown for three values of the extinction rate $\lambda: \lambda=0.01$ (solid diamonds and curve), $\lambda=0.003$ (open circles and curve), and $\lambda=0.001$ (solid triangles and curve).

question. $\tilde{p}$ would be the expected new allele frequency if there were no selection. Incorporating the effect of selection gives $p=(1+s) \tilde{p} /(1+s \tilde{p})$.

Figure 1 compares fixation probabilities estimated by simulation to theoretical predictions for different numbers of founders $k$ with $N=100, D=100, s=$ $10^{-3}, m=0.01$, and various values of $\lambda$. In all cases the predictions are very close to the simulation results, differing by no more than $2.6 \%$. Predictions of mean times to fixation are also close to observed values (within $1.7 \%$; results not shown).

Tendency for a common deme of origin: In these simulations founders have some tendency to come from the same deme, characterized by the parameter $\phi$. This tendency modifies what happens after extinction. With probability $\phi$, the founders come from the same deme. A source deme is chosen at random. The allele frequency in this source is then the probability parameter for a binomial random variable. With probability $1-$ $\phi$, the founders are chosen independently from the population at large and the probability parameter is $\bar{x}$.

Figure 2 compares predicted and observed fixation probabilities over a range of $\phi$ values for different values of $\lambda$ with $k=4, N=100, D=100, s=10^{-3}$, and $m=$ 0.01 . The predictions agree well with the simulation results (within 5.7\%). Mean times to fixation are also predicted well (within 2.2\%; results not shown).

Dominance or local frequency dependence: These simulations involve frequency-dependent selection in a haploid population. They can also be interpreted in 


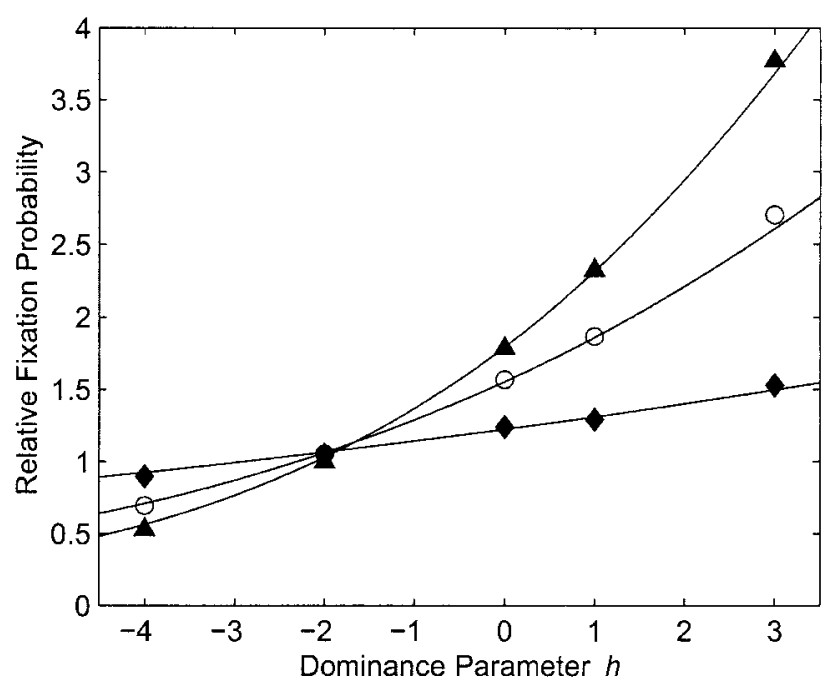

FIGURE 3.-Predicted and observed relative fixation probabilities in the presence of local frequency dependence or dominance. In all cases $N=100, D=100, m=0.01, s=$ $10^{-4}$, and the $A$ allele was initially present in a single copy. Simulation results and predictions as functions of the dominance parameter $h$ are shown for three values of the extinction rate $\lambda: \lambda=0.01$ (solid diamonds and curve), $\lambda=0.003$ (open circles and curve), and $\lambda=0.001$ (solid triangles and curve).

terms of dominance in a diploid population, and I use the parameterization involving $s$ and $h$ to describe the pattern of selection. In all of these simulations the number of founders was one. Selection differed from that described above only in that the selection coefficient $s$ was replaced by the marginal selection coeffiecient $\hat{s}$, so that $p=(1+\hat{s}(\tilde{p})) \tilde{p} /(1+\hat{s}(\tilde{p}) \tilde{p})$.

Figure 3 shows predicted and observed relative fixation probabilities for a range of values of the dominance parameter $h$ for three different values of $\lambda$ with $k=1$, $N=100, D=100, s=10^{-4}$, and $m=0.01$. The predictions agree well with the observed values: With one exception, all of the predictions are within $3.5 \%$ of the simulation results (the prediction for $\lambda=0.001$ and $h=-4$ differs from the simulation result by $6.4 \%$ ). This figure illustrates that the dependence of fixation probability on both $h$ and $\lambda$ is captured well by the analytic results. Figure 4 shows that mean times to fixation are also predicted well by the theory (within $2.2 \%$ ).

\section{DISCUSSION}

The results presented here describe the fates of alleles under selection in a subdivided population with local extinction and subsequent recolonization by an arbitrary number of founding alleles. In the simplest case treated, selection is genic and frequency independent and founding alleles have no tendency to come from the same subpopulation. These results were extended to cases where founding alleles come from the same deme with any specified probability. They were also

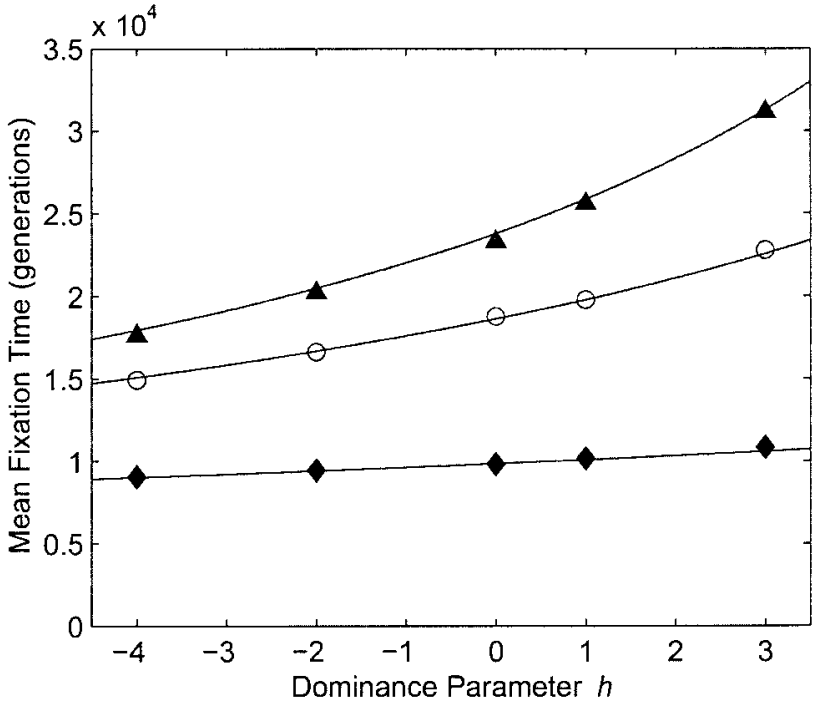

Figure 4.-Predicted and observed mean times to fixation in the presence of local frequency dependence or dominance. The plotted points represent the same simulation runs presented in Figure 3.

extended to cover dominance for fitness or frequency dependence.

In all of the cases analyzed, the diffusion approximation for the subdivided population was identical to that for a panmictic population with a different size and different selection parameters. This fact allowed the definition of effective values of population-genetic parameters: the effective population size $N_{\mathrm{e}}$, the effective selection coefficient $s_{\mathrm{e}}$, and, in the case of dominance, the effective dominance parameter $h_{\mathrm{e}}$. These effective parameters are equal to the actual parameters that characterize a hypothetical equivalent panmictic population. Substitution of effective for actual parameters in classical results for panmictic populations allows prediction of such quantities as fixation probabilities and expected times to fixation.

Computer simulations confirm that the theoretical results give a good description of the trajectory of allele frequency under the conditions modeled. Probabilities of fixation are predicted to within a few percent, as are mean times to fixation. Figure 1 demonstrates that the theory captures the effect of multiple founders. Figure 2 shows that a tendency for founders to come from the same deme is properly accounted for. Figures 3 and 4 illustrate that predictions are good for cases of dominance or frequency-dependent selection.

The effect of extinction and recolonization varies with the (effective) number of founders and their tendency for a common deme of origin. $F_{\mathrm{ST}}$ can be either raised or lowered by extinction and recolonization. This is evident from Equation 21. It can also be seen from consideration of two extreme cases. If recolonization involves a singe haploid founder, the allele frequency in the deme immediately goes to zero or one, which 
corresponds to maximum local differentiation from the population average. This leads to higher $F_{\mathrm{ST}}$. If, on the other hand, there is a very large number of founders, which have no tendency to come from the same deme, then an extinction/recolonization event moves the local allele frequency to approximately the population mean. This destroys local differentiation and lowers $F_{\text {ST }}$.

The product $N_{\mathrm{e}} s_{\mathrm{e}}$, which determines fixation probability, is also affected by extinction and recolonization. Despite the fact that $F_{\mathrm{ST}}$ can be either raised or lowered by extinction and recolonization, the direction of the effect on $N_{\mathrm{e}} s_{\mathrm{e}}$ does not depend on the pattern of recolonization: $\left|N_{\mathrm{e}} s_{\mathrm{e}}\right|$ is always lowered by extinction and recolonization, as is clear from Equation 20. The change in $F_{\mathrm{ST}}$, whatever its direction, has the same effect on the mean change in allele frequency due to selection and the component of the variance due to ordinary genetic drift. Extinction/recolonization events are always a source of additional variance, but no additional directional change. Thus the magnitude of the ratio of the mean to the variance, which is given by $\left|N_{\mathrm{e}} s_{\mathrm{e}}\right|$, is decreased by extinction and recolonization, and in its presence selection is made effectively weaker relative to stochastic change.

These results extend the situations in which a subdivided population with selection can be related to a roughly equivalent panmictic population. This equivalence allows the application of a wealth of established results for panmictic populations (e.g., KIMURA 1957; Kimura and OHтA 1969) to the subdivided populations.

\section{LITERATURE CITED}

BARTON, N. H., 1993 The probability of fixation of a favoured allele in a subdivided population. Genet. Res. 62: 149-157.

Cherry, J. L., 2003a Selection in a subdivided population with dominance or local frequency dependence. Genetics 163: 1511-1518.
Cherry, J. L., 2003b Selection in a subdivided population with local extinction and recolonization. Genetics 164: 789-795.

Cherry, J. L., and J. WAKeley, 2003 A diffusion approximation for selection and drift in a subdivided population. Genetics 163: $421-428$.

Dobzhansky, T., and S. Wright, 1941 Genetics of natural populations. V. Relations between mutation rate and accumulation of lethals in populations of Drosophila pseudoobscura. Genetics 26: $23-51$.

Kimura, M., 1957 Some problems of stochastic processes in genetics. Ann. Math. Stat. 28: 882-901.

Kimura, M., and T. OHTA, 1969 The average number of generations until fixation of a mutant gene in a finite population. Genetics 61: 763-771.

LANDE, R., 1985 The fixation of chromosomal rearrangements in a subdivided population with local extinction and colonization. Heredity 54 (3): 323-332.

Maruyama, T., 1970 On the fixation probability of mutant genes in a subdivided population. Genet. Res. 15: 221-225.

Maruyama, T., 1974 A simple proof that certain quantities are independent of the geographical structure of population. Theor. Popul. Biol. 5: 148-154.

Maruyama, T., and M. Kimura, 1980 Genetic variability and effective population size when local extinction and recolonization of subpopulations are frequent. Proc. Natl. Acad. Sci. USA 77: $6710-6714$.

Roze, D., and F. Rousset, 2003 Selection and drift in subdivided populations: a straightforward method for deriving diffusion approximations and applications involving dominance, selfing and local extinctions. Genetics 165: 2153-2166.

Slatkin, M., 1977 Gene flow and genetic drift in a species subject to frequent local extinctions. Theor. Popul. Biol. 12: 253-262.

Slatkin, M., 1981 Fixation probabilities and fixation times in a subdivided population. Evolution 35: 477-488.

Spirito, F., M. Rizzoni and C. Rossi, 1993 The establishment of underdominant chromosomal rearrangements in multi-deme systems with local extinction and colonization. Theor. Popul. Biol. 44: 80-94.

Whiтlock, M. C., 2002 Selection, load and inbreeding depression in a large metapopulation. Genetics 160: 1191-1202.

Whitlock, M. C., 2003 Fixation probability and time in subdivided populations. Genetics 164: 767-779.

Whitlock, M. C., and N. H. Barton, 1997 The effective size of a subdivided population. Genetics 146: 427-441.

WrIGHT, S., 1940 Breeding structure of populations in relation to speciation. Am. Nat. 74: 232-248.

Communicating editor: M. A. Asmussen 